\title{
Feasibility of Non-thermal Plasma Assisted Semiconductor Material Synthesis for Thermoelectric, Photovoltaic and Energy Applications
}

\author{
A. R. H. Rigit, M. U. Mushtaq, K. Sanaullah, A. Khan, and W. Javid
}

\begin{abstract}
Silicon being the major component in the semiconductor industry has proved itself very effective in myriad applications that include its use in transistors, energy storage, photovoltaics etc. A boom into its efficiency has emerged with the use of the Nano technology, when the same material is used at Nano scale for the fabrication of the devices in the said applications, it provides promising characteristics. Yet the synthesis techniques for the usage of the silicon as material at Nano scale encounter myriad hurdles, however, still the application of non-thermal plasma at Nano scale has found promising results in this field. It could bring about a huge improvement into its efficiency and can thus become ideal for the vast industrial uses. Yet it encounters several issues that makes this practice economically not feasible, like it needs more time to process such material rather than the conventional methods to synthesize materials on the liquid, solid or gas phases have their own short comings which will result into certain compromises the synthesizers have to deal with based on the properties of the end finished product. On the contras, Non-thermal plasma processing is found cost effective with properties like lack of agglomeration and more efficiency.
\end{abstract}

Index Terms-Nano crystals, silicon, synthesizers, photovoltaics.

\section{INTRODUCTION}

Silicon belongs to group IV of the periodic table with its place at the 4th group it is half way up in between the metals and halogens. 4 electrons in its valence shell makes it ideal for the use to the semi-conductor industry where the important applications of it involve the use of the silicon in energy storage devices, transistor manufacturing and photovoltaics etc. Still there is provision of the improvement into the said material it is being investigated that if the synthesis of the silicon is processed at Nano scale, it could bring about a huge improvement into its efficiency and can

Manuscript received September 9, 2018; revised January 18, 2019

A. R. H. Rigit is with the Department of Mechanical \& Manufacturing Engineering, University Malaysia Sarawak (UNIMAS), 94300, Kota Samarahan, Sarawak, Malaysia (e-mail: arigit@unimas.my).

M. U. Mushtaq and K. Sanaullah are with the Faculty of Engineering, Department of Chemical Engineering \& Energy Sustainability, University Malaysia Sarawak (UNIMAS), 94300, Kota Samarahan, Sarawak, Malaysia (e-mail: muhammadumarmushtaq@gmail.com, skhairuddin@unimas.my).

A. Khan is with the Faculty of Engineering, Department of Mechanical \& Manufacturing Engineering, University Malaysia Sarawak (UNIMAS), 94300, Kota Samarahan, Sarawak, Malaysia (e-mail: afrasyabunimas@gmail.com).

W. Javid is with the Department of Mechanical Engineering, Faculty of Mechanical and Aeronautical Engineering, University of Engineering and Technology (UET), 47050, Taxila, Punjab, Pakistan (e-mail: waqasjavid92@gmail.com). thus become ideal for the vast industrial uses. Yet it encounters several issues that makes this practice economically not feasible, like it needs more time to process such material rather than the conventional methods, particles may not be well distributed even with the use of the most effective modern techniques in this line and the prohibitive cost being needed for the establishment of this facility. The use of the non-thermal plasma for the synthesis of these particles is a new facet of this technology that has been introduced recently and has shown effective to address the above three short-comings to certain extent. The semiconductors group elements have the tendency to tune the property in them to address the targeted properties needed in them for the issues like surface coating of desired properties. Such a property can be achieved using desired discernibility into the solvent to achieve the desired end. Non-Thermal Plasma can change the chemistry of the surface by bringing the changes into the gas phase of the solvent. A visible advantage of this process can be seen in using the group IV elements coatings on the anode in the batteries to improve its stability.

\section{NANO MATERIALS CHARACTERISTICS}

Nano materials offer a very promising side of properties due to their inherent ability to be dispersed uniformly, thus establishing the uniform core of properties across all the material body. During the last decade several improves in this regard are the clear proof of this very fact that this technology has proved its effectiveness on every front [1], [2]. The usage of the semi-conductor materials synthesized and fabricated using Nano technology has not merely confined to semi-conductor applications, rather it ranges its usage in biotechnology, military applications, energy production, storage, environmental technologies and in medical applications. Being lying between the very small i.e. atomic scale and very large i.e. macro scale, Nano materials can change the properties of the same material to considerable extent. Most of the properties that include mechanical, electrical, optical and thermal can be improved if the material is synthesized and processed from bulk to Nano scale. The main change that has been brought into the properties of the material is due to the changes in the energy level and band gap, where any change into these two parameters bring change into the properties of the material that are dependent on size. Yet all the elements from group 2 till group 6 have been used in myriad technologies including the photovoltaics, light emitting diodes etc. [3]. But group 4 elements have less 
energy levels and band gaps than the rest of the elements of group 2 till 6, which make these elements most suitable for the purpose.

\section{EFFECT OF Size ON THE SEMI-CONDUCTORS PROPERTIES}

Group 4 elements of periodic table show different properties which are mainly dependent on their structures, bonding with chlorine and hydrides and oxidation states, which bring the variation within the same group from metals to non-metals. Whereas with in the same group based on the allotropic properties Tin is available in semi-conductor as well as conductor [4], [5]. Due to the strong covalent bonds in carbon it has high boiling and melting points [6]. In addition to it silicon has the most prominent place in this group with myriad applications in technological industries whereas it composes $28 \%$ of the whole earth crust [7]. Silicon shows both metallic and non-metallic properties when it's size is reduced to $4 \mathrm{~nm}$. Its optical and electrical properties have been changed a lot due to surface effects. For example, the photoluminescence intensity can be adjusted with the help of the variation in the band gap and particle size. Several efforts have been made to address the issue of the particle size using size controlling methods [9], [10]. The reduction in photoluminescence intensity due to the removal of hydrogen has resulted into the blue shift which is still a problem being prevailed [8], [9]. At different particle sizes the peak energy for silicon has different values [10] as shown in Fig. 1, where the size distribution effects the photoluminescence properties of the materials [11], [12], the presence of oxygen and nitrogen also affects the properties of the materials to a large extent [13], [14]. Since the effect of the size is directly associated to the band gap, the band shift is thus directly correlated with the particle sizes [15]. Literature shows that the silicon has the prominent optical properties out of all the elements in the group 4 [16], [17]. The dispersion of the Nano particles inside the material matrix results into the formation of the material suitable for the LEDs or photovoltaic applications [18]-[22], where the Nano crystals are the building blocks of the transistors that have prospective applications in thermoelectric, electronic, photovoltaic and batteries [23]-[28].

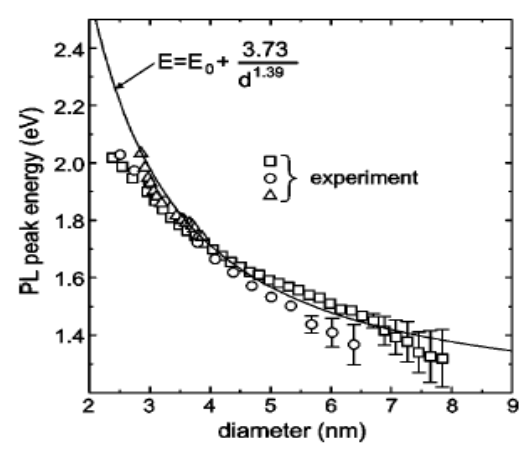

Fig. 1. Relationship between the photoluminescence peak energy and particle diameter [10].

\section{Conventional Synthesis TeChNiQUeS}

The synthesis techniques of the Nano silicon are based on the phases in which it has been synthesized. When chemical reactions take place in solvents this gives birth to the methods such as evaporation, decomposition, sol gel, and precipitation. Most of the Nano-particles belong to the elements of group 2 till 6, which have been synthesized using chemical methods, but these produce wide distribution of the particles with the low quality Nano particles, so such methods are not preferred for silicon synthesis. The reason is being the elevated temperature and pressure environment that is needed for the formation of organosilane [29].

Milling is another method that can produce Nano particles as small as $5 \mathrm{~nm}$ [29]. Gas phase techniques are useful to produce high quality Nano particles. Pulsed laser technique is another technique which can be used for the purpose where the target is a small piece of metal and laser is pulsed at femtosecond's rate, which ejects the silicon atoms out from the surface and thus forms the Nano particles with less contamination from the surface. Here, gases like argon helium etc. have been used for the treatment of the surfaces using the laser impingement. The pressure of these gases has also effect on the nature of the particles as investigated by the use of the fluid inertial model. It has been found that if the pressure of the gas has been raised, it will help in increasing the particles size and binding energy between them [30], as shown in the Fig. 2.

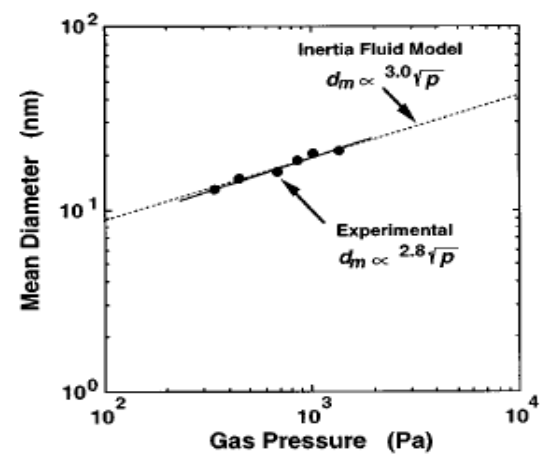

Fig. 2. Relationship between particle mean diameter and ambient gas pressure [30].

\section{NON-THERMAL Plasma SyNTHESIS OF NANO PARTICLES}

Nano particle synthesis can be conducted in three different methods that include liquid, solid and gas but each of these methods have their own draw backs. It includes:

Liquid Phase: High cost, long processing time, multiple steps involved, and large amount of solvents used.

Solid Phase: Controlling the shape and size of the nanoparticles.

Gas Phase: Require high temperatures which yield size distribution because of particle agglomeration.

Plasma is another method used for the synthesis of the Nano-particles, whereas the main advantage of using the plasma is the thin film of silicon that can be deposited on another material for the sack of modified surface properties using the deposited surface. This technique needs no high pressure or temperature, so it's cost effective. As plasma is composed of 4th state of matter so it contains itself high energy electrons and other entities [31], which can be used for the purpose of synthesis. The electric field is applied across the material with the inert gas as medium between the 
material and the electrodes. The temperature of the gas species reaches to about $200,000 \mathrm{~K}$ to $50,000 \mathrm{~K}$ before the material has been bombarded to produce the Nano particles whereas the radii of the produced particles depends on the temperature of the electrons [32]. The generation of the negative particles inside the NTP is advantageous in the sense that it depreciates the agglomeration of the Nano sized particles. Since the polymers show both conductive as well as metallic properties therefore, the use of the polymers seems favorite for being used in the electronic applications till medical sciences. Also, the reflection of light from the Silicon based Nano structures has been observed that sparks the application of this phenomena in illuminations using solid state lightening, optoelectronics and fluorescent agents for biological applications. The innovation still lacks control on production of desired results so different approaches have been adopted to attain the optimum high yield synthesis process for the desired purpose [13]. The improvements that have been brought about in this regard include surface functionalization of Silicon and Germanium Nano crystals for their application in the fields of photovoltaics and luminescent [33] along with efforts to address issues related to identification of microstructure, surface chemistry, mono-dispersity and doping [34]. On germanium side the effort to control the size of the nanocrystal results into promising achievement as it has been found that the size can be controlled via modifications in the residence time of the crystals within the plasma environment [35]. Similar efforts can be found elsewhere in literature for Si [36] as well as for sub-10nm, core shell, and carbon-coated crystalline Si (c-Si) Nano particles for energy related applications [37]. Around 3-15nm sized crystals have been synthesized at $\mathrm{H}_{2}$ concentration of around 5-70\% and pressure of 1-12 Torr with major dependence of the size is being on the residence time [38]. The use of the FTIR spectroscopy and thermal effusion have been made to synthesize 3-5 nm particles of crystalline and amorphous Silicon (Si). It has been found that at higher power of plasma Si particles have been crystalized in gas phase [39].

Plasma synthesized in-situ organic functionalization of hydrogen terminated silicon Nano crystals have been performed using total reflection FTIR spectroscopy. It has been observed that while doing hydrosilylation of Si Nano crystals the paths of alkenes and alkynes are different, which have proved useful in surface ligand passivation [40]. Some reviews papers and thesis on research findings related to non-thermal plasma synthesis of Si Nano crystals for photovoltaic, solar and other related applications are very useful in this regard [41]-[45].

\section{Plasma Polymerization}

The formation of a polymer layer under the condition prevailed inside the Non-thermal plasma reactor is called Plasma Polymerization. There are two types of reactors used for the purpose, one is bell Jar and the other is tubular type reactors. In bell jar two parallel plates have been excited using the DC current whereas in the case of the tubular reactor, Radio Frequency (RF) source is coupled with the power source [46]. The properties of the material thus produced are related to the conditions prevailed inside the reactor [47], whereas the distance between electrodes, electrode sizes and power are the three parameters which are the determining factors for the particle sizes as compared to the power and the flow rate [48], [49]. The main advantage of using the Non-thermal plasma source is the reduction in the processing time which has been achieved using plasma processing of the materials for Nano particle synthesis and thus producing materials with enhanced properties.

\section{CONCLUSION}

Materials show enhanced properties when their particle sizes change from bulk to Nano scale. Nano scale material synthesis provides added advantage due to the uniform size distribution as well as better surface properties due to lower energy gap and band gaps. To synthesize materials on the liquid, solid or gas phases have their own short comings which will result into certain compromises the synthesizers have to deal with based on the properties of the end finished product. On the contras, Non-thermal plasma processing is found cost effective with properties like lack of agglomeration and more efficiency. Further improvements can be brought into the processed material if provided with the more conductive Nano particles layer in the plasma polymer and further investigations can be conducted on the issues of the mechanical stability of the added layer. These studies could help to find the ways through which stresses can be reduced inside the material being used as coatings on the electrodes in the batteries.

\section{ACKNOWLEDGMENT}

The authors of this paper are thankful to Government of Malaysia through Ministry of Education and University of Malaysia Sarawak (UNIMAS) which support this work through grants FRGS [Grant Reference No: FRGS/TK03(03)/1303/2015(20)].

\section{REFERENCES}

[1] M. A. Reed, R. T. Bate, K. Bradshaw, W. M. Duncan, W. R. Frensley, J. W. Lee, and H. D. Shih, "Spatial quantization in GaAs--AlGaAs multiple quantum dots,” Journal of Vacuum Science \& Technology B: Microelectronics Processing and Phenomena, vol. 4, no. 1, pp. 358-360, 1986.

[2] S. Schmitt-Rink, D. A. B. Miller, and D. S. Chemla, "Theory of the linear and nonlinear optical properties of semiconductor micro crystallites,” Physical Review B, vol. 35, 1987.

[3] E. Hao, H. Sun, Z. Zhou, J. Liu, B. Yang, and J. Shen, "Synthesis and optical properties of CdSe and CdSe/CdS nanoparticles," Chemistry of Materials, vol. 11, pp. 3096-3102, 1999.

[4] J. O. Bird and P. J. Chivers, "Newnes engineering and physical science pocket book,” Newnes Pocket Books, 2014.

[5] P. Y. Yu and M. Cardona, Fundamentals of Semiconductors: Physics and Materials Properties, Germany: Springer, 2010.

[6] H. McArthur and D. Spalding, "Engineering materials science: properties, uses, degradation and remediation," Mechanical Engineering, vol. 126, no. 11, 2004.

[7] M. A. Sommers, Silicon, New York, America: the Rosen Publishing Group, 2007.

[8] L. T. Canham, "Silicon quantum wire array fabrication by electrochemical and chemical dissolution of wafers," Applied Physics Letters, vol. 57, pp. 1046-1048, 1990.

[9] X. Li, Y. He, and M. T. Swihart, "Surface functionalization of silicon nanoparticles produced by laser-driven pyrolysis of silane followed by HF- HNO3 etching,” Langmuir, vol. 20, pp. 4720-4727, 2004.

[10] G. Ledoux, J. Gong, F. Huisken, O. Guillois, and C. Reynaud, "Photoluminescence of size separated silicon nanocrystals: 
Confirmation of quantum confinement,” Applied Physics Letters, vol. 80, pp. 4834-4836, 2002.

[11] P. F. Trwoga, A. J. Kenyon, and C. W. Pitt, "Modeling the contribution of quantum confinement to luminescence from silicon nanoclusters," Journal of Applied Physics, vol. 83, pp. 3789-3794, 1998.

[12] C. Meier, A. Gondorf, S. Lüttjohann, A. Lorke, and H. Wiggers, "Silicon nanoparticles: Absorption, emission, and the nature of the electronic bandgap,” Journal of Applied Physics, vol. 101, 2007.

[13] L. Mangolini, E. Thimsen, and U. Kortshagen, "High-yield plasma synthesis of luminescent silicon nanocrystals," Nano Letters, vol. 5, pp. 655-659, 2005

[14] Y. Chao, S. Krishnamurthy, M. Montalti, L. H. Lie, A. Houlton, B. R. Horrocks, L. Kjeldgaard, V. R. Dhanak, M. R. C. Hunt, and L. Šiller, "Reactions and luminescence in passivated Si nanocrystallites induced by vacuum ultraviolet and soft-x-ray photons," Journal of Applied Physics, vol. 98, no. 4, 2005.

[15] T. Van Buuren, L. N. Dinh, L. L. Chase, W. J. Siekhaus, and L. J. Terminello, "Changes in the electronic properties of Si nanocrystals as a function of particle size," Physical Review Letters, vol. 80, no. 17, 1998.

[16] F. Erogbogbo, K. T. Yong, R. Hu, W. C. Law, H. Ding, C. W. Chang, P. N. Prasad, and M. T. Swihart, "Biocompatible magneto fluorescent probes: Luminescent silicon quantum dots coupled with super paramagnetic iron (III) oxide,” ACS Nano, vol. 4, pp. 5131-5138, 2010

[17] Z. Kang, Y. Liu, C. H. A. Tsang, D. D. Ma, X. Fan, N. B. Wong, and S. T. Lee, "Water-soluble silicon quantum dots with wavelength-tunable photoluminescence,” Advanced Materials, vol. 21, pp. 661-664, 2009.

[18] A. G. Cullis, L. T. Canham, and P. D. J. Calcott, "The structural and luminescence properties of porous silicon," Journal of Applied Physics, vol. 82, no. 3, pp. 909-965, 1997.

[19] T. W. Kim, C. H. Cho, B. H. Kim, and S. J. Park, "Quantum confinement effect in crystalline silicon quantum dots in silicon nitride grown using $\mathrm{SiH}_{4}$ and $\mathrm{NH}_{3}$," Applied Physics Letters, vol. 88, no. 12, 2006.

[20] D. Jurbergs and E. Rogojina, "Silicon nanocrystals with ensemble quantum yields exceeding 60\%," Applied Physics Letters, vol. 88, no. 23, 2006.

[21] K.-Y. Cheng, R. Anthony, U. R. Kortshagen, and R. J. Holmes, "High-efficiency silicon nanocrystal light-emitting devices," Nano Letters, vol. 11, no. 5, pp. 1952-1956, 2011.

[22] C.-Y. Liu, Z. C. Holman, and U. R. Kortshagen, "Optimization of Si NC/P3HT hybrid solar cells,” Advanced Functional Materials, vol. 20, no. 13, pp. 2157-2164, 2010.

[23] Y. Ding, Y. Dong, A. Bapat, J. D. Nowak, C. B. Carter, U. R. Kortshagen, and S. A. Campbell, "Single nanoparticle semiconductor devices," IEEE Transactions on Electron Devices, vol. 53, pp. 2525-2531, 2006.

[24] N. Petermann, N. Stein, G. Schierning, R. Theissmann, B. Stoib, M. S. Brandt, C. Hecht, C. Schulz, and H. Wiggers, "Plasma synthesis of nanostructures for improved thermoelectric properties," Journal of Physics D: Applied Physics, vol. 44, no. 17, 2011.

[25] J. K. Lee, K. B. Smith, C. M. Hayner, and H. H. Kung, "Silicon nanoparticles-graphene paper composites for Li ion battery anodes," Chemical Communications, vol. 46, no. 12, pp. 2025-2027, 2010.

[26] C. K. Chan, H. Peng, G. Liu, K. McIlwrath, X. F. Zhang, R. A. Huggins, and Y. Cui, "High performance lithium battery anodes using silicon nanowires," Nature Nanotechnology, vol. 3, pp. 31-35, 2008.

[27] A. I. Hochbaum, R. Chen, R. D. Delgado, W. Liang, E. C. Garnett, M. Najarian, A. Majumdar, and P. Yang, "Enhanced thermoelectric performance of rough silicon nanowires," Nature, vol. 451, pp. $163-167,2008$

[28] J. M. Spurgeon, S. W. Boettcher, M. D. Kelzenberg, B. S. Brunschwig, H. A. Atwater, and N. S. Lewis, "Flexible, polymer-supported, Si wire array photoelectrodes,” Advanced Materials, vol. 22, pp. 3277-3281, 2010.

[29] C. Lam, Y. F. Zhang, Y. H. Tang, C. S. Lee, I. Bello, and S. T. Lee, "Large-scale synthesis of ultrafine Si nanoparticles by ball milling," Journal of Crystal Growth, vol. 220, pp.466-470, 2000.

[30] T. Yoshida, S. Takeyama, Y. Yamada, and K. Mutoh, "Nanometer-sized silicon crystallites prepared by excimer laser ablation in constant pressure inert gas," Applied Physics Letters, vol. 68 no. 13, pp. 1772-1774, 1996.

[31] M. A. Lieberman and A. J. Lichtenberg, Principles of Plasma Discharges and Materials Processing, New York: John Wiley \& Sons, 2005.

[32] U. Kortshagen and U. Bhandarkar, "Modeling of particulate coagulation in low pressure plasmas," Physical Review E, vol. 60, no. 1, p. 887, 1999.
[33] U. Kortshagen, R. Anthony, R. Gresback, Z. Holman, R. Ligman, C. Y. Liu, L. Mangolini, and S. A. Campbell, "Plasma synthesis of group IV quantum dots for luminescence and photovoltaic applications," Pure and Applied Chemistry, vol. 80, pp. 1901-1908, 2008.

[34] Z. C. Holman and U. R. Kortshagen, "Plasma production of nano device-grade semiconductor nanocrystals," Journal of Physics D: Applied Physics, vol. 44, 2011.

[35] R. Gresback, Z. Holman, and U. Kortshagen, "Nonthermal plasma synthesis of size controlled, monodisperse, freestanding germanium nanocrystals,” Applied Physics Letters, vol. 91, 2007.

[36] Y. Ding, R. Yamada, R. Gresback, S. Zhou, X. Pi, and T. Nozaki, “A parametric study of non-thermal plasma synthesis of silicon nanoparticles from a chlorinated precursor," Journal of Physics D: Applied Physics, vol. 47, 2014

[37] R. P. Chaukulkar, K. de Peuter, P. Stradins, S. Pylypenko, J. P. Bell, Y. Yang, and S. Agarwal, "Single-step plasma synthesis of carbon-coated silicon nanoparticles,” ACS Applied Materials \& Interfaces, vol. 6, pp. 19026-19034, 2014

[38] R. Gresback, T. Nozaki, and K. Okazaki, "Synthesis and oxidation of luminescent silicon nanocrystals from silicon tetrachloride by very high frequency non thermal plasma," Nanotechnology, vol. 22, 2011.

[39] B. N. Jariwala, N. J. Kramer, M. C. Petcu, D. C. Bobela, M. C. M. van de Sanden, P. Stradins, C. V Ciobanu, and S. Agarwal, "Surface hydride composition of plasma-synthesized Si nanoparticles," Journal of Physical Chemistry C, vol. 115, pp. 20375-20379, 2011.

[40] B. N. Jariwala, O. S. Dewey, P. Stradins, C. V Ciobanu, and S. Agarwal, "In situ gas-phase hydrosilylation of plasma-synthesized silicon nanocrystals," ACS Applied Materials \& Interfaces, vol. 3, pp. 3033-3041, 2011.

[41] U. Kortshagen, "Nonthermal plasma synthesis of semiconductor nanocrystals," Journal of Physics D: Applied Physics, vol. 42, 2009.

[42] I. Dogan and M. van de Sanden, "Gas-phase plasma synthesis of free-standing silicon nanoparticles for future energy applications," Plasma Processes and Polymers, vol. 13, pp. 19-53, 2016.

[43] R. Skinner, "Sintering plasma-synthesized silicon germanium nanocrystals," M.S. thesis, University of Minnesota, the United States, 2016.

[44] A. Abduly and A. Jubran, "Fundamental and applied studies of non-thermal plasma,” Ph.D. dissertation, Newcastle University, United Kingdom, 2016

[45] V. Dollen and P. Martin, "Sodium Flux Growth of Bulk Gallium Nitride,” Ph.D. dissertation, University of California, Santa Barbara, the United States, 2016.

[46] H. Biederman and D. Slavinská, "Plasma polymer films and their future prospects,” Surface and Coatings Technology, vol. 125, pp. 371-376, 2000.

[47] K. Kanamura, Y. Kawai, S. Yonezawa, and Z. Takehara, "Diffusion behavior of anions in polyaniline during electrochemical undoping. 2. Effect of the preparation conditions of polyaniline on the diffusion coefficient of BF4," Journal of Physical Chemistry, vol. 98, pp. 2174-2179, 1994.

[48] H. Yasuda, Plasma Polymerization, Orlando: Academic Press, 1985.

[49] X. Gong, L. Dai, A. W. H. Mau, and H. J. Griesser, "Plasma-polymerized polyaniline films: Synthesis and characterization,” Journal of Polymer Science Part A: Polymer Chemistry, vol. 36, pp. 633-643, 1998.

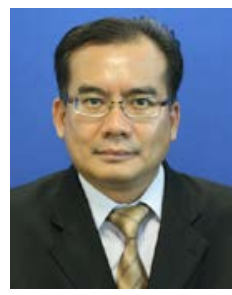

A. R. H. Rigit is from Kuching, Sarawak. He obtained his bachelor degree in mechanical engineering from the City University, London in 1995, master degree from Universiti Malaysia Sarawak in 1997, and Ph.D. from Imperial College London in 2004. He is now a professor in mechanical engineering at Universiti Malaysia Sarawak, lecturing and supervising fluid mechanics and energy related subjects and a registered professional engineer with Board of Engineers, Malaysia.

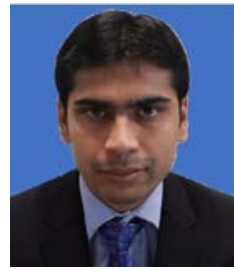

M. U. Mushtaq is from Attock, Pakistan. He obtained his bachelor degree in chemical engineering from Wah Engineering College, University of Wah, Pakistan in 2015 and master degree in chemical engineering with specialization in fluid dynamics from Universiti Malaysia Sarawak (UNIMAS), Malaysia in 2018. He is a registered engineer with Pakistan Engineering Council (PEC). 


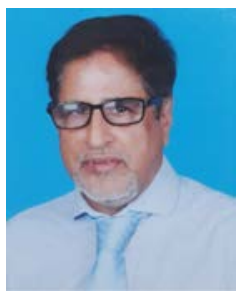

K. Sanaullah gained an extensive experience and knowledge in multiphase dynamics, gained through his Ph.D. from Birmingham University in 1995 and postdoctoral research projects funded by BP, AEA Technology, PowerGen, Fords etc. over 12 years in UK from 1990 to 2002. The period from 2002 to 2010 was devoted to gaining both academic and administrative experience in which he served positions as Lecturer, Assistant Professor and Assoc.

Professor in U.K, China and Pakistan. During this period, he served National University of Science \& Technology (NUST), Pakistan whereas Head of Department of Chemical Engineering he served a monumental role to launch the School of Chemical and Material Engineering. During his present tenure in University Malaysia Sarawak (UNIMAS) since 2011, here apart from contributing effectively \& improving undergraduate program in Chemical Engineering, his attention has gone to contribute and consolidate MS/Ph.D program in the Dept. of Chem. Engg. \& Energy Sustainability. He led Fluid Transport and Separation Group, which has served for the development of research capabilities in the areas such as Micro Channel Technology, Multiphase flows and instrumentation as well as industrial waste water treatment using, alternative energy (e.g. fuel grade ethanol, biodiesel etc.) and Plasma discharge in liquids. He was in a position to secure 6 research grants as principal investigator, including one international grant, Newton Research Collaboration Programme (NRCP) and could manage to publish $20+$ publications with over $80 \%$ published in ISI indexed impact factor journals.

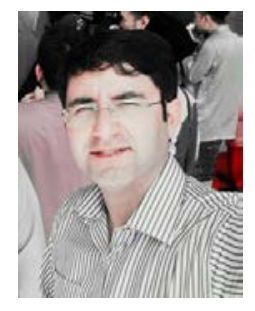

A. Khan has done M.Sc. in physics followed by M.S. in energetic materials engineering, major subjects studied were combustion and thrust related studies, and he has completed my Ph.D from University Malaysia Sarawak (UNIMAS). Before this, he has served in a scientific organization for a period of more than 7 years where he has extensively worked on the projects which aimed to understand the physics of the flow related phenomena and on multiphase flow diagnostics. His objectives are always to put into practice the broader spectrum of physics in engineering and on fluid flow related studies and specially to apply the state of the art instrumentation and diagnostic techniques for exploring the in depth realities of the physical phenomena prevailed for various multiphase fluids systems. He always wishes to widen his understanding and knowledge regarding fluid mechanics. However, he also wishes to develop novel techniques by using his knowledge. His research interests mainly are multiphase flows, compressible flows, computational fluid dynamics, mechanical designing and manufacturing and experimental techniques in fluids flow characterization.

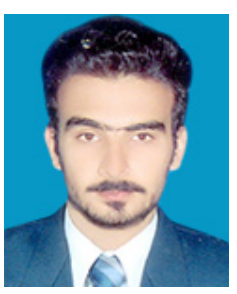

W. Javid is from Chakwal, Pakistan. He obtained his bachelor degree in mechanical engineering from Wah Engineering College, University of Wah, Pakistan in 2015 and master degree in mechanical engineering with specialization in thermal engineering from University of Engineering and Technology (UET), Taxila, Pakistan. He is a registered engineer with Pakistan Engineering Council (PEC). 